Trieste

\title{
IL NATALE NELLE LINGUE SLAVE
}

Nel presente articolo 1 autrice cerca di delineare una rassegna di nomi per 1 avvento per la vigilia di Natale, e per il Natale nelle lingue slave. Una siffatta analisi lessicale ed etimologica non può però essere fine a se stessa, ma attinge anche ad altre discipline, quali ad es. la storia della Chiesa, la storia delle religioni, e soprattutto la storia delle tradizioni popolari, che possono svelare, per una delle maggiori feste della cristianità, ancora oggi la inaspettata sopravvivenza, o meglio convivenza, di simboli pagani e cristiani. A volte il discorso si allarga necessariamente anche alle usanze, alle credenze popolari ed ai termini delle feste di altri popoli europei. Si è tentato infine di delineare almeno per $i$ termini designanti il Natale 1 area di diffusione e la relativa cronologia.

Sembra strano, eppure ci vollero tre secoli, prima che la Chiesa celebrasse ufficialmente la festa in onore della nascita di Cristo. Ancora oggi non siamo del tutto sicuri se essa venne introdotta nel. 336 oppure nel $354 \mathrm{~d} . \mathrm{Cr}$. L 'unico dato sicuro pervenutoci a questo proposito riguardala costruzione della cappella della Natività nel 360 d. Cr. per ordine di papa Liberio nella chiesa che dal IX sec. in poi fu nota, in Roma, come la basilica di S. Maria Maggiore. La celebrazione della festa del Natale incontrò all inizio anche 1 opposizione di alitorità insigni quali origene e s. Agostino, che vi vedevano dei residui di culti nagani. Soprattutto in Oriente essa trovò molte difficoltà ad affermarsi, a Bisanzio fu celebrata per la prima volta nel 397, in Egitto venne introdotta appena verso 430 , mentre la Chiesa armena non la conosce neppure oggi.

Nella Chiesa orientale, si riusci a risolvere le controversie spostando la festività al 6 gennaio, giorno dell Epifania, che veniva celebrata con grande lustro, coincidendo $i$ festeggiamenti con quelli in onore di Dioniso, dio del vino e protettore della fertilità. Più tardi, quando 
anche in Oriente la festa del Natale si rese indipendente da quella dell 'Epifania, quest "ultima conservò maggior splendore grazie alla tradizione del culto dionisiaco.

Infine, quando dopo lunghe controversie si fissò la data della nascita di Cristo alla notte fra il 24 e $i 125$ dicembre, anche questa non fu da parte della Chiesa una decisione occasionale, ma motivata dalla presenza alla stessa data di un 'altra festa pagana, quella del Dies Natalis Solis Invicti in onore del dio Mitra (di origine orientale), il culto del quale si diffuse in Roma in forma misterica soltanto nel I sec. $d_{\text {. }} \mathrm{Cr}$., sebbene la figura del dio sia antichissima e risalga molto probabilmente al periodo dell unità indo-iranica ( $\theta$. KeZzner, Heortologie oder die geschichtliche Entwicklung des Kirchenjahres und der Heiligenfeste, Freiburg 1906, p. 94-104).

Il nome di Mitra compare già nei più antichi testi sacri della religione indiana, i Veda, e nell-Avesta, libro sacro dei Persiani, e vi è sempre rappresentato come dio del cielo. A Roma il culto del dio si differenzia dagli altri culti misterici, come p.es. quelli di Osiride, Cibele, Dioniso, Demetra, tutti di origine agraria e miranti all unione del fedele col dio su questa terra, poichè il seguace di Mitra non propone di unirsi al dio, ma soltanto di assicurarsi la divinità come guida della propria anima attraverso le sette sfere planetarie al1 'empireo. In seguito, nel III-IV sec. d.Cr., 1 -importanza che $i 1$ dio Mitra assunse nel mondo romano fu tale da essere identificato col sole. Anzi in alcuni bassorilievi esso venne raffigurato staccato dal sole, ed il sole stesso ha nei suoi confronti addirittura la funzione dell iniziando (Le grandi religioni, Rizzoli, Milano 1964, p. 558-567).

Ques to breve excursus sulle origini del culto di Mitra potrebbe apparire al primo momento ozioso ai fini di questa ricerca, ma ad un analisi più approfondita esso si rivela quanto mai necessario, per capire il sostrato pagano su cui si innesto la festa del Natale e contro cui dovette combattere a lungo la Chiesa per cancellarne le tracce piu evidenti. Il periodo natalizio venne infatti a coincidere per espresso volere della Chiesa con $i$ festeggiamenti pagani del solstizio invernale, di cui quelii in onore di Mitra erano appunto la manifestazione più trasparente. $\grave{E}$ 
chiaro quindi che la Chiesa volle contrapporre ad un mito cosi diffuso e radicato, di cui 1 espressione piùsfolgorante er a 11 Dies Natalis Solis Invicti, la festa del Natale, che, a prescindere dalla Pasqua, è indubbiamente 1a più importante nel mondo religioso cristiano.

De1 resto fin dalla più remota antichità nel1 ultima decade di dicembre ed a11 inizio di gennaio $i$ popoli indoeuropei cercavano di propiziarsi il ritorno del Sole, simbolo della vita e della fecondita. Specialmente nelle regioni nordiche, dove la notte si protraeva per lunghi mesi, gli indigeni tentavano di svegliare la natura assopita attraverso il culto del fuoco. Il residuo più palese di questa antichissima tradizione si è conservato fin quasi ai giorni nostri attraverso la tradizione del ceppo natalizio, che veniva acceso la vigilia di Natale e spento appena col finire delle feste, $i l$ giorno dell Epifania. Quest'uso era diffuso in una buona parte del territorio europeo e presso certi popoli, come avremo occasione di vedere in seguito, $i 1$ ceppo di Natale finì per dare 11 nome al giorno stesso della vigilia. ${ }^{1)}$

Il periodo natalizio inizia con 1 avvento, periodo durante il quale la Chiesa si prepara all arrivo del Signore (come lo indica del resto la stessa parola latina adventus) con liturgia speciale ed almeno anticamente, agli albori del Cristianesimo ed attraverso tutto il Medio Evo, con un rigoroso digiuno, del quale sono rimaste tracce evidenti nella terminologia della Chiesa orientale. Analizzando il termine per 1 avvento nelle lingue slave potremo osservare come $i$ popoli dell area cattolica abbiano preso di peso per la designazione di questo periodo il termine latino adventus (p.es. s1. advènt, cr. àdvenat, c. advent, slov. advent, pol. advert, sor. sup. adwent, sor. inf. adwent) oppure abbiano coniato un calco come sor. sup. přikhad, i1 sor. inf. psichod ${ }^{2}$, 1'a. pol. przyjście ( si confronti per quest 'ultimo termine $E$. Frankowski, Kalendarz obrzedowy ludu polskiego, Warszawa 1928, 62) ed il sbcr. prišašce, došas̀ce? (Gospòanje) (Skok, I, 10). Si tratta indubbiamente di calchi diretti dal latino, non passati cioè attraverso la mediazione del tedesco, poichè ai tempi della cristianizzazione degli slavi anche $i l$ clero germanico adoperava come base dell insegnamento $i$ testi latini, cercando di renderli comprensibili alle popolazioni analfabete nelle loro rispettive parlate. La 
liturgia era poi esclusivamente quella latina, quindi era abbastanza naturale che nel tradurre il termine liturgico adventus in mezzo alle popolazioni slave si cercasse, in mancanza di un termine equivalente già esistente, di renderlo comprensibile con un calco.

I popoli di religione ortodossa preferiscono invece ricordare il digiuno, e nella maggior parte dei casi si servono di espressioni quali "digiuno natalizio", così gli Slavi orientali usano le espressioni $r$. rożéstvenskij post, ucr. mizdvjănyj pist, bruss. kaljadny post, bulg. kòledni pòsti, mac. božikni/božik'ni posti, s. bözićni post, formate tutte da un sostantivo panslavo derivante da $11^{\circ}$ a.sl.eccl. postz, a sua volta prestito da11 aat. fasto "digiuno" (Miklosich, SEW, 260, Kiparsky, 261, Vasmer II, 415-416) e da aggettivi derivanti da voci che designano i] Natale.

In polacco, un altro termine interessante per designare l'avvento è i] sostantivo composto przedgody, < prep. przed "davanti" + dial. gody "Natale" (v. sotto).

$L$ ucraino e il bielorusso conoscono inoltre un "espressione che al primo momento potrebbe sembrare un po'esotica e precisamente ucr. pylýpivka e bruss. pizipaǔka, che si riferisce però semplicemente al giorno di s. Filippo (14 novembre), con il quale nella Chiesa orientale inizia 1 avvento, due settimane prima rispetto alla Chiesa cattolica. In pratica 1 avvento degli ortodossi dura così sei settimane, riflettendo in questo modo usi antichissimi, risalenti addirittura al $\mathrm{V} \mathrm{sec}$., quando $i 1$ digiuno natalizio veniva definito quadragesima sancti Philippi (N. Nizles, Kalendarium manuale utriusque ecclesiae orientalis et occidentalis, Oeniponte 1896 [Pars I: Festa immobilia], 330). Un residuo di questa antica tradizione la si trova nell antico polacco, dove czterdziestnica oltre ad avere i] significato vero e proprio di "quaresima" veniva altresì ad indicare le sei settimane del1 avvento, prima che questi cominciasse ad essere osservato il giorno di s. Caterina (25 novembre) (cf. Frankowski, z. c.). Non vi è alcun dubbio che, come nel caso di przyjscie, anche questo termine polacco sia un. calco diretto dal latino.

Il giorno di s. Filippo viene indicato dagli Slavi meridionali, e precisamente Serbi e Macedoni, con un altro termine interessante, bòżióne pokzade, rispettivamente božikni/ bożikni pokladi:pòklade/pokladi sono 
composti dal prefisso po- e dalla radice *kLo- $d-/ k 2 \bar{\alpha}-d-$ "stendere, apporre" (cf. lit. klöti, kloju "stendere", paklõde "lenzuolo" (Brückner SEJP, 236, e Skok II, 91), ed indicano 1 'ultimo giorno prima del digiuno natalizio, giorno in cui non si mangia ancora di magro, bensì si preparano, "si appongono, si distribuiscono le vivande". In serbo si usa tra 1'altro 1'espressione bële pòklade per indicare la Dominica in Sexagesima, la domenica cioè che precede quella del Carnevale, chiamata dagli Ortodossi Domenica déi Zatticini, poichè 1 'ultima in cui si può mangiare $i 1$ formaggio, $i 1$ burro, ed $i$ prodotti caseari, nonchè mésne pòklade per designare la Dominica in Quinquagesima, la domenica di carnevale, 1 "ultima in cui si possa mangiare la carne.

Sia detto per inciso che ai termini bèle e mésne pòkzade corrisponde nelle località di Risan in Dalmazia 1 'espressione Hristovi zâuzi (Vuk, 207-208), termine quest 'ultimo che risale all'a. sl. eccl. vezati (cf. c. vázati, pol. wiazać, russo vjazát6) "legare", cioè in senso traslato "proibire", e trova un corrispondente puntuale nel rum. cîmeleağ $<$ carne $(m)$ liga(t) $\left(3^{0}\right.$ pers. sing. ind. pres. di Zigare "legare" e cisşlegi (p1. di cişleagă), con cui si proibisce non più la carne ma il cacio (rum. cass) (Tagliavini, 211 e Skok III, 584).

Anche in bulgaro il giorno di s. Filippo viene indicato con un termine simile al s. pòklade, e cioè kòzedni zàgovezni "ultimo giorno prima del digiuno, quando si può ancora mangiare grasso" (RSBE I, 367). Zàgove-

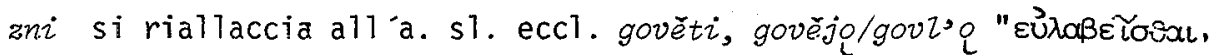

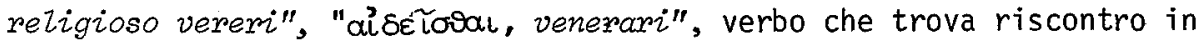
quasi tutte le lingue slave, conservando un significato simile al1 "a. sl. eccl. nello s1. dial. goveti (Slavia Veneta) "osservare un rispettoso silenzio, digiunare", r. govét6, ucr. hovíty, bruss. havéc6 "digiunare" (> lit. gavéti, lett. gavêt "digiunare"), mentre in alcune altre $c$. hovět, slov. hoviet', sor. sup. (arc.) howié e sbcr, gòvjeti è passato al significato di "favorire, assecondare, soddisfare" (Bezlaj I, 165). Anche $i 1$ bulgaro conosce $i 1$ verbo goveja nel significato "osservare un rispettoso silenzio, digiunare" (Bezlaj, 2., c., Georgiev BER I, 258), ma sembra che nel termine zàgovezni sia confluito sia il significato del digiuno sia quello di "assecondare, soddisfare la voglia di mangiar bene, 
mangiar grasso" prima del.1ungo digiuno dell avvento.

Passiamo ora in rassegna $i$ termini de 1 giorno che precede la festa del Natale, la vigilia, giorno in cui $i$ fedeli si preparano ad accogliere la nascita del Signore con rigoroso digiuno e, appunto, la vigilia, che si protrae fin dopo la messa di mezzanotte.

Lo sloveno dialettale del Goriziano conosce per la vigilia di Natale un termine che trae origine dal lat. vigizia, vigizare e precisamente bîlja. Si riscontra però tale voce anche in altre parti della Slovenia, poichè la troviamo registrata dal Megiser (fine sec. XVI) biza "digiuno", e dal Gutsmann (fine sec. XVIII) sveta biza "vigilia di Natale". Si tratta di un prestito dal latino, ma attraverso la mediazione del1 "ant. bav. vilge "vigilia di festa" (Bezlaj I, 21 e Striedter-Temps, 91). Direttamente dal latino deriva invece vîjja, diffuso nel litorale, nei dintorni di Trieste, che trova un riscontro puntuale nel polacco wilja (accanto a wigizia).

Sempre all idea del vegliare e molto probabilmente alla messa mattutina del Natale si riferisce 7 'interessante vocabolo amanais della alle del Torre, metatesi di a madins "al mattino". Tale espressione viene però alternata anche con božiéna: vîjja ${ }^{3)}$.

Diffuso esclusivamente nella Slavia meridionale è invece un termine, legato al culto del fuoco, accennato poc'anzi, ed a 11 'uso di accendere alla vigilia di Natale il cosiddetto ceppo natalizio, che secondo credenze precristiane era propiziatore di forza vitale e simbolo del sole, ed accanto al quale venivano scaldarsi le anime dei defunti antenati. Tale termine, sbcr. bàdnjak, mac. badnik, bulg. bĭdnik, s1. badnik/badnjak (diffuso esclusivamente sul confine croato, nella regione della Bela Krajina, e solo eccezionalmente nella Carniola Inferiore [Dolenjska] nelle forme bâdni večêr e bâdni dân) viene ad indicare sia la vigilia di Natale come 10 stesso ceppo natalizio e si ricollega all a. sl. eccl. bढděti "vigilare, vegliare" (Bezlaj I, 8).

Sarà interessante rilevare come il suddetto termine si sia diffuso anche nel gruppo di dialetti romeni, parlati nella regione nord-orientale di Salonicco (specialmente nellavalle di Meglena), dove era ancora viva al1 inizio di questo secolo la forma boadnic nel significato di "ceppo natalizio" e di "vigilia di Natale". (I. Mareu, Quelques aspects 
des anciennes coutûmes dihiver dans la vallée de Moglena, MK, VIII (1975), 70).

Nello sloveno, il ceppo di Natale viene definito anche bož乞̌nik e božiž con $i l$ nome stesso del Natale (nel Goriziano) oppure čồ (nel Litorale), prestito dal friulano zoc, e ěrija (nel Collio goriziano) (W. Kuret, Praznično leto Slovencev IV, Celje 1970, 77-109) da rapportare a ěuiti, čijjem "sentire, udire, custodire", verbo di diffusione panslava senza un parallelo corrispondente nel gruppo baltico, da collegare alla radice ie. *keu- "sentire, osservare". (Skok I, 345).

L'usanza di bruciare un grosso ceppo di legno per Natale è largamente diffusa in territorio europeo, così p. es. in Toscana troviamo per il Natale la denominazione Pasqua di ceppo (o semplicemente iz ceppo) dal latino cippus, "pietra di confine, palo". Interessante è qui 1 "uso della parola Pasqua, che dalla denominazione di una speciale festa ebraica passò a designare nella terminologia cristiana un altra festa ben determinata, la quale, per estensione di significato, divenne sinonimo di "festa" in generale (si confronti a questo proposito il termine spagnolo Pascuas de Navidad, Tagliavini, 182).

Del resto anche $i$ Tedeschi conoscono il ceppo di Natale, il JuZbZock, la cui designazione trae origine da una voce per il Natale, Juz, diffusa soprattutto nella Germania nordorientale, strettamente affine alle parole che indicano il Natale nelle lingue scandinave (svedese, danese, norvegese juz, islandese $j o z$ ) e presente anche in anglosassone (geohhoz, geóla, "Natale"), in inglese yule "feste natalizie", probabilmente prestito dalle lingue scandinave, ed in gotico. In quest "ultima lingua in un antico calendario troviamo attestato fmua jiuleis "novembre", letteralmente "primo Nata1e", il che farebbe supporre un *aftuma jiuzeis "dicembre". Sia detto per inciso che dalle lingue germaniche settentrionali la voce è passata anche nelle lingue ugrofinniche: finnico jouzu, estone jouz, lappone juovza "Natale". L'etimologia di questa parola non è chiara, probabilmente risale ad una forma germanica *jehwza "tempo delle tempeste di neve", ma in ogni modo è certo che si tratta di un termine precristiano che doveva indicare una festa di mezzo inverno, alla quale si sostituì, con la comparsa del Cristianesimo, quella della nascita del Redentore, pur rimanendo inalterato il vecchio nome. 
(Tagliavini, 184-185).

La Slavia occidentale, ed in parte quella orientale, definisce la sera di Natale in modo diverso: štedmy večer, slov. štedry večer, sor. sup. šcedry wječor, secondo il Miklosich (Chr. term., 24), 1 ucr. ščédryj vécirs (accanto a dóbryj e bogátyj véčir) ed inoltre il bruss. šcadréc ${ }^{4)}$. In polacco szczodry wieczor indica invece la sera di s. Silvestro e quella che precede 1 Epifania (Brückner SEJP, 545). Si tratta in questo caso di un aggettivo protoslavo * šedrz $<{ }^{*}$ sked- "spargere"+suffisso -rz (cf. gr. onebauvuu "diffondere, spargere" dal significato "munifico, generoso" (Brückner, 1. c.).

Lo sloveno e 1 ucraino conoscono inoltre l'espressione svêti večêr rispettivamente svjatyjj véčir "sera santa".

Per indicare la vigilia di Natale il polacco ed il sor. inf. si servono di un "espressione singolare gwiazd(k)a e gwězd(k)a "stella (stellina)" che $i$ ragazzini portano in giro per le case del paese, adornata ed illuminata, cantando canti natalizi. Anticamente anche 10 slovacco designava la vigilia con un espressione simile: dohviezdny večer (Miklosich, Chr. term., Z. c.).

I] russo a sua volta conosce un altro termine interessante che trae origine dal cibo che veniva preparato la notte di Natale: socél6nik, da collegare (Vasmer II, 704) a sóčenc, "una specie di focaccia" e questo a sua volta al termine panslavo *sokr "succo" (cf. lit. sakat (pl.) "resina", gr. Z̈rós, con psilosi ionica $<$ *ónós "succo", termini tutti derivanti dall ie. *sok" os, Frisk, s. v.).

Dal nome del cibo rituale che si prepara in questo giorno traggono origine le denominazioni per la vigilia di Natale in ucr. kutjá e bruss. kuccja. Si tratta di un cibo speciale preparato col grano, papavero e miele, diffuso in gran parte del territorio slavo, come 10 comprovano 1'a. s1. eccl. kutija/kucija (Supr. 120, 30; 122, 11), a. russ. kutijai kuigja, r. kutijia, pol. kutia/kucja (in Mickiewicz nel poema Dziady kucyja) ed il bulg. dial. kučá, dove il termine non indica la pietanza natalizia, ma bensi il cibo che si depone sulle tombe, per onorare i morti (Stawski SEJP III, 444).

Dal bielorusso si è diffuso nelle lingue baltiche: lit. kūǔià "cibo 
speciale per la vigilia di Natale", "vigilia di Natale", kũě̃u vãkaras "sera di Natale", küěiu naktis "notte di Natale" (Kurschat, II 1232) e lett. kükes "cibo preparato per la vigilia di Natale", küku diena "il 24 dicembre", küku vakars "sera del 24 dicembre".

Allo stesso gruppo di parole dovrebbe appartenere anche lo slov. kuckriih, un pane speciale che veniva cotto la vigilia di Natale nella Stiria orientale, farcito di frutta secca, specialmente pere e noci, con 1 intento di allontanare gli influssi malefici dalla casa, sebbene il Berneker (I, 654) vi si opponga decisamente.

L'origine' di tutte queste voci non è sicura. Sembrerebbero derivare dal ngr. xouxxi (pl. XoinuLó) "fava". (Berneker e Sławski, 2. c.).

Per quanto riguarda la vigilia di Natale in Ucraina la kutjá viene definita "ricca" (bagátaja), mentre era "povera". o letteralmente "di fame" (golódnaja) la kutjä per la vigilia dell "Epifania.

I Bielorussi a loro volta conoscono tre giorni con questo stesso nome: vjaltkaja o pèršaja kuccjá ("grande" o "prima") per Natale, quando il digiuno è molto rigoroso e sono proibiti sia la carne sia i latticini, bagátaja kucejá "ricca" per la vigilia di Capodanno, poichè si mangia di tutto e si può anche bere, ed infine bédnaja ("misera") o apóšnjaja ("u1tima") kucejá per la vigilia dell Epifania, durante la quale si mangiano solo latticini ${ }^{5)}$.

Citiamo infine un termine interessantissimo, che ha suscitato non pochi problemi etimologici. Si tratta del sor. sup. patoržica, sor. inf. (dial.) paterżyca ${ }^{6)}$, che è stato brillantemente spiegato dallo SchusterŠewc (ZfSI I, 1956, 26-29). Secondo 10 studioso il termine sarebbe da scomporre in un prefisso $p a-+1$ la radice torz- $/\left(\right.$ torg $\left.^{-}\right)+i 1$ suffisso $-i c a . \mathrm{Da} 1$ punto di vista semantico il prefisso pa- avrebbe un senso negativo come p. es. nel sor. pa-duch, c. pa-douch "ladro", sor. pa-kluě, c. pa-kziě "grimaldel10". La radice torž- (torg-) avrebbe $i 1$ significato di "mercato, commercio" e sarebbe da rapportare al protosl. *tzrgz "mercato" (cf. r. torg, pol. torg, c. trh). -erm nella forma dialettale pateržyca sarebbe invece riflesso di $r$ nel sorabo inferiore. Il termine verrebbe così a significare un giorno poco adatto al mercato, al commercio, come 10 erano in Germania secondo antiche tradizioni medioevali la vigilia di Natale, 
11 giorno di s. Silvestro, Mercoledì delle Ceneri ed il Venerdì Santo (Schuster-šewc 2. c.).

Passiamo ora all analisi dei termini veri e propri per il Natale.

Ne11 a. s1. eccl. riscontriamo il termine Rožd6stvo Christovo (Ostr.) ${ }^{7}$ ), calco sul greco ñ révunous tỡ Xolotoũ "Natale" (letteralmente "nascita di Cristo") o anche sul bizantino tò Xolotoúrevva (termine che vive fino ad oggi nel neoellenico). Dalla voce antico slava sono sorte le denominazioni ufficiali per il Natale presso gli Slavi di religione ortodossa: r. Roždestvo, ucr. Rizdvo, bruss. Rastvó, bulg. Roždestvò, mac. Rożdestvo, s. Rożdèstvo, la maggior parte delle quali è rimasta relegata al 1 inguaggio strettamente liturgico, mentre soltanto in russo ed in ucraino il termine suddetto è confluito nel linguaggio popolare.

Nella Chiesa cattolica il termine ufficiale per il Natale è Nativitas Christi, da cui è sorta tutta una serie di calchi presso gli slavi cattolici: pot. Boże Narodzenie, 1 a.c. Narozenie Pána (XIV sec.), c. mod. Narozeni páně, lo slov. Božie narodenie, s\}. Božje rojstvo, Kmistovo rójstvo, le forme croate Rojstvo, Rodstvo (XIII s.), Roštvo (XIV e XV s.) Hristovo, Bǒžje (Skok III, 152). Probabilmente un calco dal latino è anche $i 1$ bruss. Naradżènne. Tutti questi termini, a prescindere dal polacco Boże Narodzenie, che si è diffuso anche nel linguaggio popolare, sono di uso prettamente ecclesiastico. ${ }^{8}$ )

Dal termine protoslavo *Kolęda, prestito dal 1at. KaZendae trae origine tutta una serie di termini che indicano non solo la festa di Natale, ma anche una serie di usanze, connesse con il periodo natalizio, specialmente $i$ canti natalizi. Come abbiamo appena accennato all inizio di questo articolo, nei primi tempi del Cristianesimo la data della celebrazione della Natività di Cristo non era stata ben fissata ed in Oriente all inizio il Natale era stato festeggiato addirittura il 6 gennaio, il giorno stesso del1 Epifania; niente di strano dunque che Katendae abbia designato $i$ giorni immediatamente precedenti il Natale (overossia 1 'Epifania) ed in seguito il giorno stesso del Natale.

Fra le lingue slave il significato della festa di Natale traspare nel bulg. Kòleda (RSBE I, 261) nel bruss. Kaljády/Koljady (TSBM II, 595), e nel mac. dial. Koleda. (W. Fedorowicz, JP XLVI/5, 359). Il r. koljadá indica "il tempo natalizio, da Natale all Epifania" (Vasmer I, 606) come 
del resto 1 'espressione dial. sl. kolêdni svétki, caratteristica di tutto i1 territorio del1'01tremura (Prekmurje) (Pleteršnik I,422 e $\mathrm{M}^{9}$ )). I1 Pleteršrik (l. c.) riporta inoltre 1 'espressione kolêdnjak "mese di dicembre".

Si discostano invece dal significato di "Natale" 1"a. sl. eccl. Kolęda "Capodanno", lo sl. koléda, il pol. kolęda, l'a.c. kolada/koleda e lo slov. koleda/kol' $a d a$, che indicano la particolare tradizione di girare per il paese di casa in casa cantando canzoni natalizie. (Berneker I, 544 e W. Fedorowicz . c. c.). L'ucr. kolajäá viene usato sia nel significato di "Natale" come in quello di "canto natalizio" (Grinčenko II, 274).

Continuando nella nostra rassegna delle denominazioni popolari per $i 1$ Natale riscontriamo un "espressione diffusa esclusivamente in una parte della Slavia occidentale e precisamente nei dialetti polacchi, nel sorabo e nel ceco: *Gody> pol. dial. Gody (Karłowicz, SGP I, 906), sor. sup. Hody, sor. inf. Gody e 1 'espressione ceca Hod bożí vanoční) (Sławski I, 307). Il sorabo inf. conosce inoltre le espressioni Gódownica (si cf. però anche il sor. sup. Hodownička, a. c. Hodovnice) e gódojski swězeń nel significato specifico di "giorno di Natale". Inoltre sia nel sor. superiore che in quello inferiore $\mathbf{i l}$ nome per la festa di Natale ha dato il nome al mese di dicembre: sor. sup. hodownik, sor. inf. gódownik (cf. nel medesimo significato anche $i 1$ casciubo gózdnik, Tagliavini, 175.)

Il termine godr è panslavo, compare quindi in tutte le lingue slave, con significati però diversi: "anno, ora, tempo favorevole, festa, ecc.", nel significato di "Natale" si limita comunque all "area della Slavia occidentale summenzionata. ${ }^{10)}$

Una nota a parte merita $i 1$ termine ceco Vánoce (a. c. Vánocẽ), nonchè lo slovacco vianoce, voci completamente isolate in territorio slavo. La maggior parte degli studiosi 10 considerava un composto ibrido, un antichissimo prestito dal mat. Wînnachten, la cui prima parte sarebbe stata un prestito vero e proprio dell "agg. wr̂ch "santo", la seconda una traduzione di Nacht (cf. e. gr.. Miklosich, Chr. term., 23). Tale opinione è stata messa completamente fuori discussione da una proposta del Mareš (Slavia, 28 [1959], 516-518), accettata pure dal Machek $^{2}$ s. v., secondo il quale il c. Vónoce (ant. Vớnocē) sarebbe un prestito dal mat. Wînnachten in tutti $i$ suoi elementi: $i 1$ gruppo $-\hat{\imath} n$ - sarebbe passato $>\hat{\varepsilon}$, la seconda 


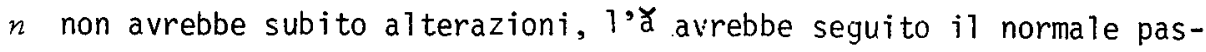
saggio $>0$, caratteristico delle lingue slave. Il tedesco $h$ veniva di solito recepito nelle lingue slave come ch ( $p$. es. ĥs-chyša), ma il gruppo cht non era consonn al sistema fonematico slavo, per cui venne sostituito con $k t$, passato regolarmente nello slavo occidentale $>c$. La desinenza -en a sua volta sarebbe passata ad e propria del nom. pl. dei temi in $-i \bar{a}$. Il mat. Winnachten sarebbe quindi passato nello slavo a *venokte e da questa forma all'a. c. Vánocẽ, c. Vánoce, slov. Vianoce. Secondo il Mareš il prestito sarebbe avvenuto nel periodo in cui esistevano ancora le nasali e prima del definitivo passaggio di $\mathbf{a}>0$, in una epoca cioè anteriore alla missione di s. Cirillo e Metodio. Sempre secondo i] Mareš sarebbe di secondaria importanza il fatto che Vánocé non compaia nello slavo ecclesiastico e negli antichi testi cechi di carattere liturgico, poichè si tratterebbe di un termine popolare di antica data, radicatosi profondamente nelle lingue ceca e slovacca fino ai nostri giorni.

Un prestito $\mathrm{A} \rightarrow \mathrm{l}$ medesimo mat. Winnachten $10 \mathrm{si}$ trova anche nei dialetti sloveni in un ampia fascia di territorio, che abbraccia tutta la Carinzia, cominciandọ da oriente nella forma BienaXte (Brdo, Podklošter, Rekarja

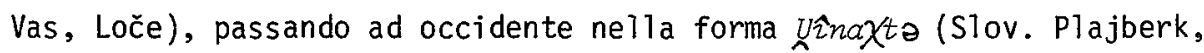
Djekše, Globasnica), scendendo quindi nella Val di Resia: Vinnate (Bela, Stolbica). Vinaxto (Osojane, Njiva) e nella valle del Torre: Vienahtif Viemahti, facendo quindi anche la sua comparsa nella Carniola Superiore (Gorenjska) nella forma Uinahti, alternante con quella della lingua letteraria $B \delta: i \check{c}^{11)}$.

Per quanto riguarda le forme resiane esse sono state annotate da Baudouin de Courtenay (Materialy dlja južnoslavjanskoj dialektologiji i etnografii, S. Peterburg 1875, 907): Vinaghti, dudu Vinahat. Tra 1'a1tro da esse trae origine anche il nome del mese di dicembre vienahtnik, attestato nel Gutsmann, e mísee ti od vinahti (per quest ultima forma cf. Milko Matičetov, Per la conoscenza degli elementi tedeschi nel dialetto sloveno di Resia [Festschrift Denison Graz 1975], 136).

Comunque nello sloveno la forma di gran lunga più diffusa è quella letteraria Bóżič, che nei dialetti del Litorale e del Goriziano appare più frequente nella forma plurale Božiči indicando così non solo la festa di Natale, ma tutto il periodo natalizio, includendo anche le altre due festività, quella del Capodanno e dell Epifania. Dal nome del Natale 
traggono origine anche le denominazioni (ormai già cadute in disuso) di due mesi, dicembre, che compare sia nella forma božǎ̌nik e božǐ̌njak, anche vêliki božžrnjak, e gennaio, mâli bož̃̂̌njak, che sono passate anche in territorio kajkavo: bòzićnjak/velikobòzićnjak' "dicembre", mentre 1'isola di Krk (Veglia) conosce:la forma (anche questa ormai desueta) bòzić$n i$ (si cf. anche $\mathbf{i}$ termini.ungheresi per "gennaio": kis karácson e "dicembre": nagy-karácson). (Pleteršnik I, 47, Skok I, 180, Miklosich, Monatsn. 22).

A proposito di quest "ultima voce sarà da rilevare che è diffusa esclusivamente nella Slavia.meridionale: oltre al summenzionato s]. Bóżč conosciamo infatti il sbcr. Bòżic, il bulg. dial. Böžič ed il mac. Božik/ Božik' (W. Fedorowicz, z. c.). Non vi è alcun dubbio che il termine sia un diminutivo (ipocoristico) di un termine pansiavo bogr "Dio" ed è appunto nel significato di "piccolo Dio" che esso compare nelle lingue slave occidentali: a. pol. bożye, attestato nel Canto dezza Bogurodzica il più vecchio canto ecclesiastico della Polonia (attribuito a s. Adaiberto) e risalente probabilmente al XIV sec., nell'a. c. božic e sor. inf. božyck (A. Brückner, Mitologia slava, [Traduzione dal polacco e note di Julia Dicksteinówna] Bologna 1923, 118).

L'etimo di bogz ha suscitato parecchie discusioni, ma sostanzialmente non vi dovrebbero essere dubbi circa la sua parentelá con 1 'ai. bhágah"signore, elargitore" ed av. baya- "signore, dio". È quasi sicuramente da escludere che sia un prestito dall 'iranico, dovuto alla mediazione degli Sciti. Tra l altro nella loro mitologia non vi è traccia alcuna di un simile nome (Brückner SEJP, 34) ${ }^{12}$ ).

$V i$ è ancora un termine nelle lingue slave che designa il Natale, molto difficile dal punto di vista etimologico, e cioè 1 'a. russo koroüunz "solstizio d'inverno", che compare nella Cronaca di Novgorod nell a. 1143 e trova corrispondenza in gran parte delle lingue slave: r. (dial.) karaěún, koročún "solstizio invernale", però anche "12 dicembre" e addiritura "morte, fine", bruss. karačun "morte improvvisa, demone che abbrevia la vita", ucr. Kračun (accanto a Krečún, Kerečun) "Natale", bulg. kračon, kračùnee "un giorno del periodo natalizio", slov. or. Kračún. "Natale" (si cf. per tutte queste voci Berneker I, 603-604) e mac. dial. Kračun (W. Fedorowicz, t. c.). 
Esso appare anche nello sloveno e nel serbocroato, con significato però del tutto diverso: sl. kračûn "catenaccio", kračôn "pezzo di legno" (Plet. I, 453), sbcr. (čak.) kràcěun "catenaccio" (a Cherso), accanto allo antrop. Kračun e il top. Kračunište (Skok II, 175). Nel significato di Natale la voce è presente anche nel1 ungh. Karácsony, probabilmente prestito dallo slavo, e nel rom. Cräciun.

Molti studiosi hanno tentato di far derivare le forme slave dal romeno, altri la forma romena dallo slavo. Già il Miklosich però (Die slavischen Elemente in Rumunischen, Wien 1861, 26) si rese conto che il rom. Crăciun doveva derivare dallo slavo essendovi un argomento fondamentale in favore di una simile origine, 1 attestazione cioè del1'a. russ. koročuinz nella cronaca di Novgorod della metà del XII sec. Vengono così a cadere tutte le etimologie latine (del resto alquanto difficili e fantasiose) del termine Crăciun. Ne enumereremo qui almeno le principali: 1) > Christi ieiunium (Schuchardt, Literaturblatt für germ. u. rom. Philologie VII [1866], 154 ess.)

2 ) $>$ ereationem (Densusianu, Istoria limbei si literaturei romane, Iaşi $\left.1894^{2}, 111\right)$.

3) > calationem (i1 lat. calatio, -onis designerebbe "la chiamata che il sacerdote faceva all "inizio del mese", Papahagi in Convorbiri Literare XXXVII (1904), 670-672.

$4)$ *quartum ieiunium, etimo del tutto artificioso proposto dal vaillant (Prilozi za književnost, istoriju i folk1or, XXIV [1958], 72, 75.)

D’altra parte non convince nemmeno la connessione di kračun slavo con le voci di tipo kračati "camminare" (per quanto accolta dal Vasmer I, 633) né quella con 1 "aggettivo *kortzkg "breve" (si v. a questo proposito Berneker I, 604).

Un 'ipotesi ardita, completamente diversa dalle altre, sull origine del rum. Crăciun, è stata fatta da E. Çabei (Studi şi Cercetări Lingvistice XII [1961], 313-317), secondo i1 quale $i 1$ senso primitivo di Crăciun non sarebbe quello di "Natale", bensi di "ceppo", data 1 "usanza, originariamente pagana, del ceppo di Natale, alla quale si è accennato all inizio del presente articolo. Seara Crăciunului sarebbe dunque come 1 albanese nata e buzmit, propriamente "la sera del ceppo" e poi. "la sera del Natale". La voce rumena potrebbe essere un antichissimo prestito dal1 albanese 
kërcuni "ceppo, zocco di legno". L“ipotesi sarebbe più attendibile, se esistesse in rumeno un crŏciun con il senso di "ceppo", come in albanese. Inoltre, secondo il Tagliavini (514), una simile proposta sarebbe difficile anche dal punto di vista fonetico, poichè un affricata dentale albanese $(c=t s)$ viene resa in rumeno di solito con $t$, , anzichè con c.

L'etimo del10 s1. kračûn e del·sbcr. kràčun, "pezzo di legno" è invece del tutto diversa. Secondo lo Skok (II, 175), sarebbe un prestito dal latino characium<gr. xapóuıov, "pa10". Le voci avrebbero potuto significare anche "ceppo di Natale", ma un simile passaggio semantico non è attestato, al contrario del casciubo, dove riscontriamo un interessante parallelo semantico fra gådka "catenaccio di legno" e godë "periodo fra Natale e Capodanno", anticamente "Natale"13).

Questi slittamenti di significato che non si limitano esclusivamente al casciubo, ma che abbiamo osservato sopra anche per il romeno, per 1 'albanese, per 1 italiano ed il croato ( $\mathrm{si}$ ricordi per queste due ultime Tingue $i$ termini Pasqua de $z$ ceppo e badnjak) sono testimoni dello antichissimo culto pagano del fuoco, di origine indoeuropea, di cui si è parlato all'inizio del presente articolo. Purtroppo, per la voce slava kračun la questione etimologica resta per $i l$ momento ancora aperta.

occorre menzionare per ultimo ancora un termine, limitato esclusivamente al polabo e cioè Trébě < protos1. *Terby (Mikiosich, Chr. term., 24) che doveva originariamente designare qualche festa pagana. Tale voce sarebbe da collegare probabilmente con il sostantivo s1. eccl. treba "sacrificio" e con $i 1$ corrispondente verbo streebiti "sacrificare, compiere una funzione religiosa" (W. Fedorowicz, 2 . c.).

A conclusione di questo articolo cercheremo di dare un quadro riassuntivo dei termini trattati, di delineare cioè 1 'area di diffusione $e$ tracciarne, per quanto possibile, i rapporti cronologici.

I termini per la festa trattata possono dividersi in otto gruppi:

1) calchi sul greco ì réurnoıs toũ Xolotoũ

2) calchi sul latino Nativitas Christi.

3) derivanti dal protoslavo *KoZeda.

4) derivanti dal protoslavo ${ }^{\star} G o d z$.

5) derivanti dal protoslavo *Bozitj6. 
6) termini del tipo Kračun.

7) prestiti dal mat. Wînnachten.

8) il polabo Trébè.

I termini dei primi due gruppi sono diffusi in tutto il territorio slavo e sono, a parte alcune rare eccezioni ( $p$. es. Roždestvó, ucr. Rizdvó, pol. Boże Narodzenie), di uso esclusiavmente liturgico. Le voci di tutti gli altri gruppi sono di origine popolare ed appartengono alla lingua letteraria, oppure ai dialetti. Estesi a tutta la Slavia orientale con ramificazioni al sud nel bulgaro e nel macedone, verso ovest nello slovacco, sono $i$ termini del tipo kračun. Molto diffuse sono le voci derivanti da *Godz, poichè si estendono in tutta la Slavia occidentale, mentre quelle derivanti da *Koleda col significato vero e proprio di "Natale" si limitano al bulgaro e al bielorusso. Tutti gli altri termini restano limitati più o meno a singole lingue.

Molto più difficile è delineare i rapporti cronologici che intercorrono fra questi gruppi di termini, poichè scarseggiano $i$ dati cronologici ed al limite si possono stabilire delle cronologie relative soltanto

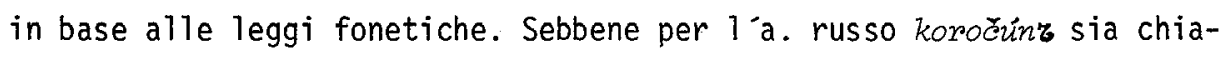
ramente attestata la data della sua prima comparsa (nella Cronaca di Novgorod del 1143), la sua origine e quella dei corrispettivi termini nelle altre lingue slave, è indubbiamente assai più antica, essendo questo termine probabilmente legato ad antichi culti pagani del fuoco, per cui 10 si può quasi sicuramente definire $i 1$ più antico fra $i$ nomi elencati. Indubbiamente molto antichi sono $i$ termini derivanti dal protoslavo *Kolęda, antico prestito dal lat. Kalendae. L'a. c. Vánocě, prestito dal mat. Wînnachten, è databile al periodo in cui non erano ancora scomparse le vocali nasali e prima che si fosse concluso il passaggio $\breve{a}=0$, nella prima metà quindi del IX sec. Con relativa sicurezza si pud anche datare 10 sl. eccl. RoždGstvo, poichè citato ne1 Vangelo di Ostromir, databile alla prima metà del1'XI sec. Assai più recente è la forma Gody, attestata alla fine del XIV sec., mentre per gli altri termini non disponiamo di elementi cronologici sicuri. 
1) Parallelamente al culto del fuoco nel mondo romano si sviluppò anche quello della vegetazione che contribuì ad un'ulteriore mescolanza fra simboli pagani e cristiani. Infatti a questo arcaico culto si riallacerà 1500 anni più tardi 1 albero di Natale che potrebbe sembrare al primo momento un "usanza prettamente cristiana. Sia detto per inciso che il presepio non si riallaccia ad antichi riti pagani, bensì al periodo della Controriforma e fu diffuso preminentemente dall ordine dei Gesuiti ( per maggiori particolari si v. N. Kuret, Praznično leto Slovencev IV, Celje 1970, 77-109).

2) Per gentile informazione fornitami dal Dr. Helmut Fasske (Institut für sorbische Volksforschung, Bautzen/Budyšin, DDR) si precisa che $i$ termini sor. sup. prikhad e sor. inf. psichod sono ormai caduti completamente in disuso.

3) I due termini mi sono stati gentilmente indicati da 1 prof. Pavle Merkù nel suo materiale manoscritto per il Vocabolario storico-dialettologico-etnografico del Torre.

4) Informazione orale gentilmente fornitami da p. Costantino Maskalik della Sezione Bielorussa della Radio Vaticana

5) Anche queste interessanti denominazioni sono dovute a p. Costantino Maskalik.

6) Si cf. a questo proposito 1a proposta dello Schneeweis, in Feste und Volksbrauche der Sorben, Berlin 1953, 98, che fa derivare il termine dal lat. pastor.

7) A causa delle diverse norme ortografiche prevalse in epoche diverse nelle varie lingue slave, mi è parso più semplice scrivere tutti $i$ nomi delle feste con la maiuscola.

8) Sebbene Nativitas Christi sia la designazione ufficiale per il Natale nel latino ecclesiastico, essa ha avuto scarsa diffusione in territorio romanzo. Benchè non sia nostro proposito analizzare nel presente articolo le espressioni per il Natale nelle lingue romanze, sarà interessante notare come nella maggiore parte di. esse $i l$ termine tragga origine da11'espressione Christi natale (e. gr. it. lett. Natale, veneto Nadal, grigionese e friulano Nadal, sardo Nadale, sic. Natali, 
provenzale e catalano Nadal, port. Natal), mentre a Nativitas risale esclusivamente $10 \mathrm{sp}$. Navidad. Il francese Noël a sua volta risale ad una forma basso-latina Notalis, in cui 1\%o per a nella prima sillaba può essere dovuto a semplice dissimilazione o a contaminazione di qualche altra parola, p. es. nox (Tagliavini, 182-183).

9) $M$ indica $i 1$ materiale manoscritto per il SLA (=Slovenski lingvistični atlas), per la cui consultazione ringrazio vivamente la prof. Martina Orožen, della Facoltà di Filosofia di Lubiana.

10) Per maggiori ragguagli circa il significato della radice *god- nelle lingue slave si $v$. la tesi di dottorato di Alenka Sivic-Dular: Pomenoslovna razčlemba besedne družine iz korena *god- $v$ slovanskih jezikih - Ljubljana 1978.

11) Le forme Vienahti/Viernahti della valle del Torre mi sono state indicate dal prof. Pavle Merkù, tutte le altre sono tratte da $M$ (v. nota 9).

12) Per maggiori dettagli, e per tutte le altre ipotesi, si v. Mayrhofer, Kurzgefasstes etymologisches Wörterbuch des Altindischen, $1963 \mathrm{I}$, 457-458.

13) I due termini casciubi sono tratti dal materiale manoscritto per $1^{\text {E ESSJ }}$ (=Etimološki slovar slovenskega jezika), messomi gentilmente a disposizione dal prof. Bezlaj.

\section{ABBREVIAZIONI BIBLIOGRAFICHE}

1) LIBRI

Berneker

Bezlaj

Brückner SEJP

Frisk

Georgiev BER

Grinčenko

Karłowicz
$=$ E. Berneker, Slavisches etymologisches Wörterbuch I, II - (1906-1913-)

$=F$. Bezlaj, Etimološki slovar slovenskega jezika I, Ljub1jana 1976.

= A. Brückner, Szownik etymologiczny jezyka polskiego. Krakow 1927.

$=H$. Frisk, Griechisches etymologisches Wörterbuch, Heidelberg, 1954 e ss.

=V. Georgiev, Brzgarski etimologičon reěnik (BAN). Sofija 1962-1969.

= B. Grinčenko, SZovar " ukrains kojr movy (I-IV), Kyīv (1907-1909).

$=$ J. Karłowicz, Szownik gwar polskich (I-VI). Kraków (1900-1911). 
Kiparsky

Kurschat

Machek $^{2}$

Miklosich Chr. term.

Miklosich Monatsn.

Miklosich SEW

Pleteršnik

RSBE

Skok

Sławski SEJP

Striedter-Temps

Tagliavini

TSBM

Vasmer
$=V$. Kiparsky, Die gemeirislavischen Lehmö̈rter aus dem Germanischen. Helsinki 1934.

= A. Kurschat, Litauisch - deutsches wörterbuch (I-IV), Göttingen (19681973).

= V. Machek, EtymoZogický sZounik jazyka českého. Druhé, opravené a doplnené vydant. Praha 1968.

= F. Miklosich, Die christziche Ter minologie der slavischen sprachen. Wien 1875.

$=F$. Miklosich, Die slavischen Monatsnomen. Wien 1868.

= F. Miklosich, Etymologisches Wörter buch der slawischen Sprachen. Wien, 1886.

$=$ M. Pleteršnik, Slovensko-nemški slovar I-II, Ljubijana 1894-1895.

= Rečnik na srvremennija br Zgarski knižoven ezik (I-III) (BAN). Sofija, 19551959.

$=$ P. Skok, Etimologijski rječnik hrvatskoga izi sroskoga jezika (I-IV). Zagreb $(1971-1974)$.

= F. Stawski, Szownik etymologicany jezyka poiskiego. Kraków 1952-.

$=H$. Striedter-Temps, Deutsche Lehrwöpter im Slovenischen, Berlin 1963.

= C. Tagliavini, Storia di parole pagane e cristiane attraverso $i$ tempi. Brescia 1963.

= Tlumačalony slounik belaruskaj movy, Minsk 1977-.

= M. Vasmer, Russisches etymologisches 
Wörtexbuch (I-III), Heidelberg (19501958).

2) RIVISTE

JP

MK

Slavia

Zfs I
$=$ J'qzyk polski. Kraków.

= Makedonski folkZor. Skopje.

= Slavia. Casopis pro slovanskou fizoZogii. Praha 1922-.

= Zeitschrift für Slowistik. Berlin.

Povzetek

BOŽIC V SLOVANSKIH JEZIKIH

V članku podaja avtorica seznam imen za božično obdobje, t.j. za advent, božični večer in božič $v$ slovanskih jezikih. Leksikalna in etimološka analiza se opira na izsledke cerkvene zgodovine, zgodovine verstev, teologije, predvsem pa etnologije, ki je raziskovalcu $v$ dragoceno pomoč pri odkrivanju mešanice krščanskih in poganskih simbolov enega izmed največjih krščanskih praznikov. Kadar je potrebno, navaja avtorica pri posameznih primerih leksikalno primerjavo z ostalimi indoevropskimi jeziki. Na koncu skuša pri imenih za božič orisati areale posameznih izrazov in ugotoviti dobo njihovega nastanka. 